\title{
ARTICLE \\ Development of Superhydrophobic Polyester (Polyethylene terephthalate) Fabric for Multiple Applications
}

\author{
Subhas Ghosh ${ }^{*}$ Roopkatha Pallye \\ College of Engineering and Technology, Eastern Michigan University, Ypsilanti, Michigan, USA
}

\section{ARTICLE INFO}

Article history

Received: 6 May 2020

Accepted: 25 May 2020

Published Online: 30 October 2020

Keywords:

Polyester fabric

Contact Angle

Fluoro silane

Super hydrophobic

Stain repellant

\begin{abstract}
This study intended to develop a healthy and environmentally friendly super-hydrophobic PET polyester textile fabric using a specific Fluoro Silane finish (SHF). A novel SHF was prepared and applied on a polyester fabric using a pad-dry-cure method. The finished fabric was evaluated for the degree of hydrophobicity, durability and stain repellence. The finished fabric exhibited static water contact angle greater than $170 \mathrm{o}$ and received 90 AATCC (4 ISO) rating that is recognized as super-hydrophobicity and this property was maintained even after a 50,000-cycle abrasion test. FTIR analysis identified the characteristic peaks related to $\mathrm{Si}-\mathrm{O}-\mathrm{Si}$ and $\mathrm{C}-\mathrm{F}$ asymmetric stretching bands of the finish on the fabric indicating a robust attachment on the fabric. Finished fabric did not show any change in appearance or tactile characteristics of the fabric.
\end{abstract}

\section{Introduction}

$\mathrm{S}$ uper-hydrophobic fabric technology was evolved from biomimetic technology. This was derived from the lotus leaf phenomenon as researched by many scientists. The term "Super-hydrophobic" is related to extremely high-water repellency of a substrate. When the static water contact angle $\theta$ is greater than $150^{\circ}$, the surface is super-hydrophobic ${ }^{[1]}$. Under such condition, the water drop forms a spherical shape achieving minimum surface area because of the cohesive forces between the water molecules. As the water drops fall on the surface, they effortlessly roll off the surface without wetting it at all. Because of very low surface energy of the super- hydrophobic surface, loosely held dirt and soils get easily attached to the rolling drops and are removed. The lotus leaf is one of the best-known natural super hydrophobic surfaces, that effectively removes mud, as water drops flow over the surface. A Nano-level hydrophobic natural wax crystal was found to be present on the top of the micro bumps on a lotus leaf, which makes the lotus leaf a self-cleaning as well as a strong hydrophobic surface ${ }^{[2]}$. There are several techniques that had been used by many researchers to produce super-hydrophobic textiles. Kawai and H. Nagata ${ }^{[3]}$ had used soft lithography, X-Ray lithography, electron beam lithography, Nano sphere lithography and photolithography to develop super hydrophobic surfaces. Zhang et al ${ }^{[4]}$ and Chen et al ${ }^{[5]}$

*Corresponding Author:

Subhas Ghosh,

College of Engineering and Technology, Eastern Michigan University, Ypsilanti, Michigan, USA;

Email:sghosh@emich.edu 
used the same technique by creating silicon nano-pillar arrays. A Phase separation technique was reported to be used for the preparation of super- hydrophobic surfaces. Polizos et al ${ }^{[6]}$ obtained a hierarchical porous structure through interconnection of different dimension of pores. They used PEG Polyethylene glycol (PEG) and PDMS in $1: 1$ ratio in the phase separation to create a surface that had a water contact angle of $160^{\circ}$. Bao et al ${ }^{[7]}$ used chemical vapor deposition method, where a substrate was created in the form of non-volatile film using gaseous reactants of metal oxide nanoparticles like $\mathrm{ZnO}$, $\mathrm{Al}_{2} \mathrm{O}_{3}$ and $\mathrm{Fe}_{3} \mathrm{O}_{4}$ etc. that produced super hydrophobic fabric surfaces. Several researchers used sol-gel and other chemical to produce super-hydrophobic surfaces. Sahoo and Kandasubramanian ${ }^{[8]}$ used organic, inorganic materials and metal alkoxides for sol-gel techniques. Hufnagel's et al. ${ }^{[9]}$ produced super-hydrophobic cotton fabric using silica particles. They reported WCA was $155^{\circ}$. Ivanova and others ${ }^{[10]}$ fabricated super-hydrophobic cotton fabric by altering the fabric structure using fluoro silane (Fs) material. Xue et al ${ }^{[11]}$ used amino and epoxy functionalized silica nanoparticles along with stearic acid and $1 \mathrm{H}, 1 \mathrm{H}, 2 \mathrm{H}, 2 \mathrm{H}$-perfluorodecyl-trichlorosilane on cotton fabric to create super-hydrophobic characteristics and they claimed to achieve $170^{\circ} \mathrm{WCA}$ (Water Contact Angle). Meng et al., ${ }^{[12]}$ Das and De, Meng et al. ${ }^{[13]}$ used long chain fluorinated compounds on cotton fabric to create super-hydrophobic characteristics. All these methods produced various degrees of Super hydrophobicity on textile substrates; but the cost of production for commercially viable products having desired durability, quality and least environmental harm, still impedes mass production of such products.

In this investigation a Super-Hydrophobic and stain repellant polyester fabric was developed by synthesizing a superhydrophobic finish using a single fluoro-silane compound and applying it on the textile substrate using simple textile wet processing method. Since some fluorine compounds have been reported to have adverse effect on health this Fluorosilane was carefully selected so that it has no harmful effect to human health and environment. The US HMIS and NFPA agencies ${ }^{[14]}$ rated the chosen Fluorosilane as 0 health hazard and 0 physical hazard. Fluoro silane was used in this investigation because the fluorinated methyl groups are less reactive and possess very low surface energy due to the presence of strong C-F bond than normal hydrophobic $-\mathrm{CH}_{3}$ groups, providing very high hydrophobicity and stain repellency with creating no environmental issues.

\section{Experimental Methods}

Material: A 100\%-woven polyester (PET) dyed fabric was used. The fabric was a plain weave having a weight of $6.710 \mathrm{z} / \mathrm{yd}^{2}$ and woven from False Twist Textured yarns. The construction was $48 \times 48$, ends x picks per inch.

Chemicals: Reagent-grade solvent butanol, disodium hydrogen phosphate, $72 \%$ sulfuric acid solution, titanium tetra butoxide, and anhydrous $\mathrm{NaOH}$ pellets were obtained from Sigma Aldrich. FS (FDTES) was obtained from Matrix Scientific. All chemicals were used without further purification.

PET fabric pre-treatment: In order to generate more attaching functional groups on the polyethylene terephthalate chains so that the super-hydrophobic finish is more durable when applied on the fabric, an alkaline partial hydrolysis was conducted on the specimen fabric. Polyester affected by alkali depending on their ionic character. Only the outer surface of polyester gets affected by ionizable alkali like caustic soda $\mathrm{NaOH}$. Deesterification of polyester results in the formation of freefunctional groups at the fiber surface as seen in Figure 1, which are terminal hydroxyl and carboxyl end groups (14). After washing the fabric in a nonionic detergent at $50^{\circ} \mathrm{C}$, de-esterification was performed at $85^{\circ} \mathrm{C}$ in a water bath containing $6 \%$ sodium hydroxide, for $20 \mathrm{~min}$, in the presence of $1 \%$ titanium tetra butoxide as an initiator. The $\% \mathrm{NaOH}$, temperature and de-esterification time were determined using a designed experiment so that enough generation functional groups occur without losing any significant strength of the Fabric. The de-esterified sample was rinsed in mild $\mathrm{H}_{2} \mathrm{SO}_{4}$ solution to neutralize the fabric.

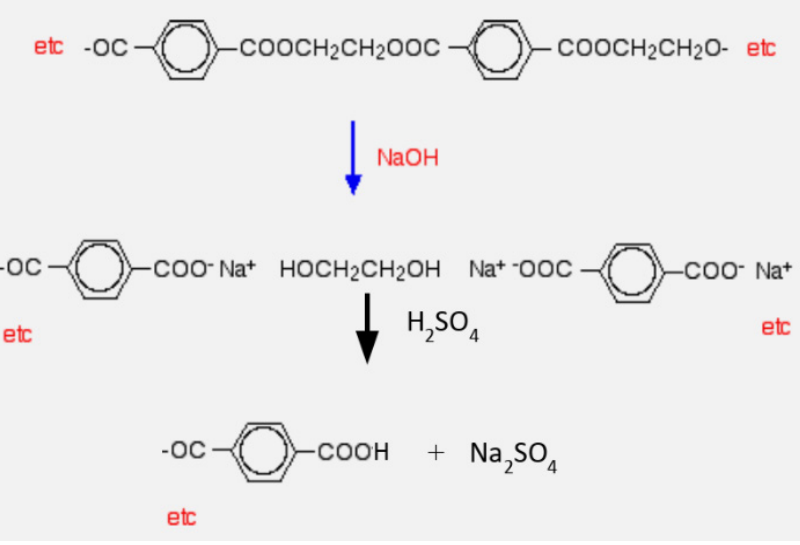

Figure 1. illustrate the PET polyester de-esterification process

Preparation of the Superhydrophobic (SHF) finish and Application: A pre-determined amount of 
Perfluorodecyltriethoxysilane (FDTES) was added to the reagent-grade solvent butanol and stirred continuously at room temperature at $350 \mathrm{RPM}$. A small amount of the catalyst, disodium hydrogen phosphate, was added to facilitate the addition of the finish to the prepared polyester specimens. A few drops of sulfuric acid were added slowly to the mixture while stirring to maintain the very low $\mathrm{pH}$ of 2 , to control the condensation reaction. The required amount of additional butanol was added and stirring was continued. The solution was stirred continuously for one hour and was placed in the refrigerator for 24 hours prior to application. Care was taken to evade rapid condensation reaction and precipitation by carefully monitoring $\mathrm{pH}$ and condensation time. The superhydrophobic finish was synthesized and then applied to the de-esterified fabric specimen using a traditional dip pad cure method. The additional finish was force sprayed onto the fabric surface prior to the fabric entering the padding mangle. This facilitated the finish to migrate into the interstices of the woven structure evenly. The fabric was dried at $60{ }^{\circ} \mathrm{C}$ for 15 minutes and then cured at $165+/-5^{\circ} \mathrm{C}$ for 15 minutes for complete attachment of the finish. These conditions were previously optimized and verified in the preliminary research.

Spectroscopic analysis by FTIR: A PerkinElmer Fourier-transform infrared (FTIR) spectrophotometer was used for spectroscopic analysis of the prepared samples. This method was used to examine the effects of deesterification on the PET fabric and application of SHF finish on the fabric.

Determination of Water Contact Angle (WCA): WCA was determined using a Static Contact Angle method. A contact angle measurement device First Ten Angstrom (FTA-200) was used for measuring static contact angle. Images for contact angle measurement were captured using an Amscope MU853B 14MP high-speed digital camera, attached to the device set up. Five readings were taken on different areas of the same sample and were analyzed by the computer plugin LBADSA available with the open-sourced JAVA application ImageJ. In the LBADSA method, the theoretical profile of the drop is not fitted to a certain drop contour but is rather optimized according to an image energy approach. It is based on the perturbation solution of the axisymmetric Laplace equation. During the fitting process, complete pixel information is used. The use of this global model results in exactly accurate contact angles. This approach chiefly has an edge over other methods when a precise accurate contour detection is difficult because of un-sharp or noisy boundaries of textile fabrics ${ }^{[15]}$.

Water repellency spray test: Superhydrophilicity of the treated fabric was evaluated for water repellency by spray test according to AATCC TM22 (ISO 4920). The test samples were rated according the rating chart provided by AATCC where a value 100 is given to the test specimen when no sticking or wetting occur on upper surface and a 0 value is given for complete wetting of upper and lower surfaces after the water spray. A substrate is recognized as superhydrophobic when a rating of 80 and higher is obtained. The fabric surface is described as wet at spray point only at 80 ratting. The results obtained from this test method are largely dependent on water repellency of the yarns, fibers, and finish on the fabric and not upon the construction of the fabric.

SHF finish durability test of the treated fabric: The durability of the finish on fabric was measured by abrasion resistance test using 50,000 cycles by ASTM method D4966-12 on a Martindale abrasion tester. Before and after abrasion, the specimens' weight losses and contact angles were measured. In addition, spectroscopic data was obtained to evaluate the effect of abrasion on finish attachment.

Stain Repellency Evaluation: Stain repellency was tested against mayonnaise, jam, and hot sauce on the fabric surface separately, and the specimens were left for 15 minutes after application of the staining substances. Then, the fabric was rinsed with normal tap water and dried. The post-washing samples were rated for stain using AATCC - 300 stain visibility chart where 5 in no stain and 0 is maximum staining of the specimens.

\section{Results and Discussion}

Free functional end groups were created by breaking the esterification links of PET chains under controlled deesterification conditions. During the de-esterification process, few long chains of the fiber polymers break down and produce larger numbers of free functional end groups such as $\mathrm{OH}-$ and COO- groups. As seen in Figures 2 and 3 a broad band associated with $\mathrm{OH}$ groups are Present at $2930 \mathrm{~cm}^{-1}$.

Furthermore $\mathrm{C}=\mathrm{O}$ band of ester linkages near $1745 \mathrm{~cm}^{-1}$ increased because of the de-esterification of PET chains and continued to increase with the $\mathrm{NaOH}$ concentration. We used $6 \%$ alkaline concentration for 15 minutes in the de-esterification process that provided enough functional groups on the PET chains. This sample was used for the application of the SHF finish because there was no significant change in the fabric strength as measured before ( $80 \mathrm{lbf})$ and after (81lbf) de-esterification. 


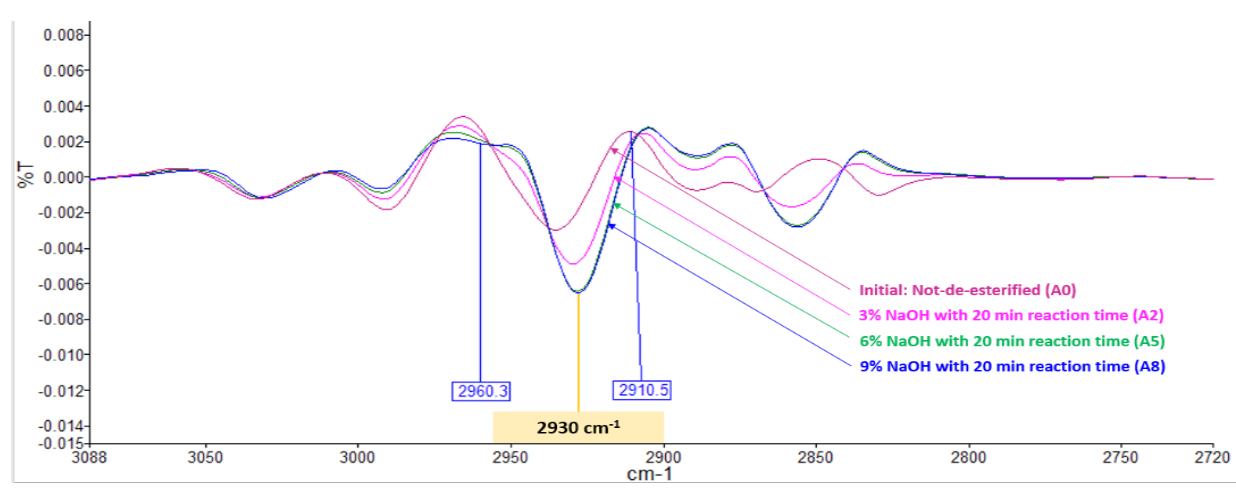

Figure 2. Second derivative transformed FTIR scans for de-esterified PET samples around the OH- bands

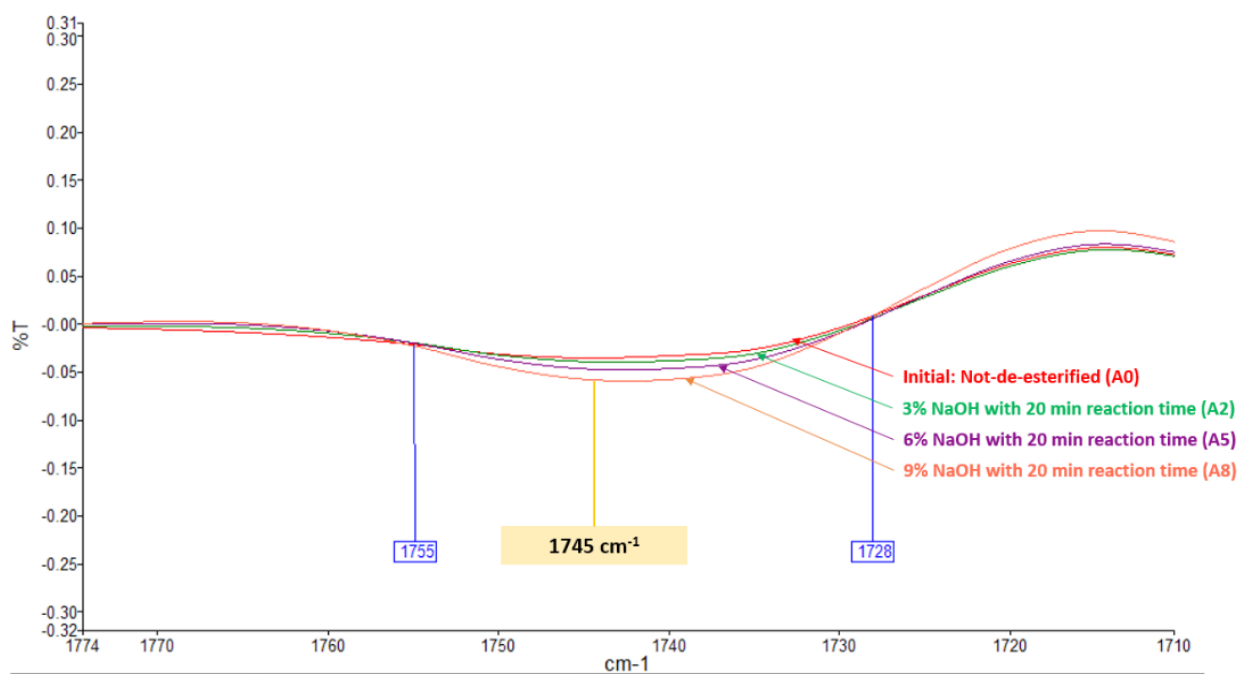

Figure 3. Second derivative transformed FTIR scans for de-esterified PET samples around the COOH- bands

De-esterified PET samples were treated with Fluorosilane finish and cured at an elevated temperature near $165^{\circ} \mathrm{C}$ resulting condensation reactions between the Fluorosilane finish and ends groups generated on the PET fabric samples thus, binding the finish on the fabric surface as illustrated in Figure 4 describing the attachment mechanism.

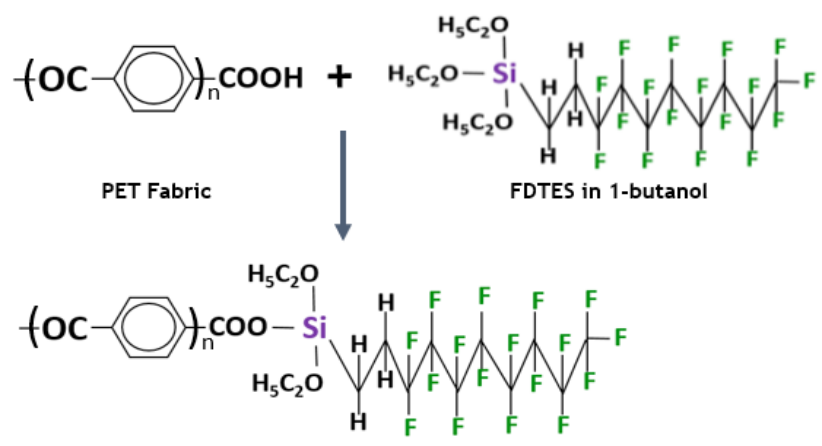

Figure 4. Shows the attachment scheme of the Fluorosilane finish on the PET fabric surface
The FTIR analysis of the Fluorosilane treated fabric sample provided a good indication about the attachment of the finish. As illustrated in Figure 5, the spectra of the treated fabric shows the peaks at $852 \mathrm{~cm}^{-1}-1094 \mathrm{~cm}^{-1}$ that are related to asymmetric stretching of Si-O-Si bonds and the picks appeared at $744.5 \mathrm{~cm}^{-1}$ and $948.88 \mathrm{~cm}^{-1}$ are assigned to the C-F bonds (16) arising from the SHF finish on the fabric. These evidences suggest that applied finish is attached to the fabric structure. Water Contact Angle (WCA) measured on the SHF finished specimen using LBADSA method, exhibited $178.2^{\circ} \mathrm{WCA}$; the same fabric specimen was subjected to 50,000 cycles of abrasion that provided $170.8^{\circ} \mathrm{WCA}$. These results reveal the strong superhydrophobic characteristic property of the fabric and the durability of its super-hydrophobicity. 


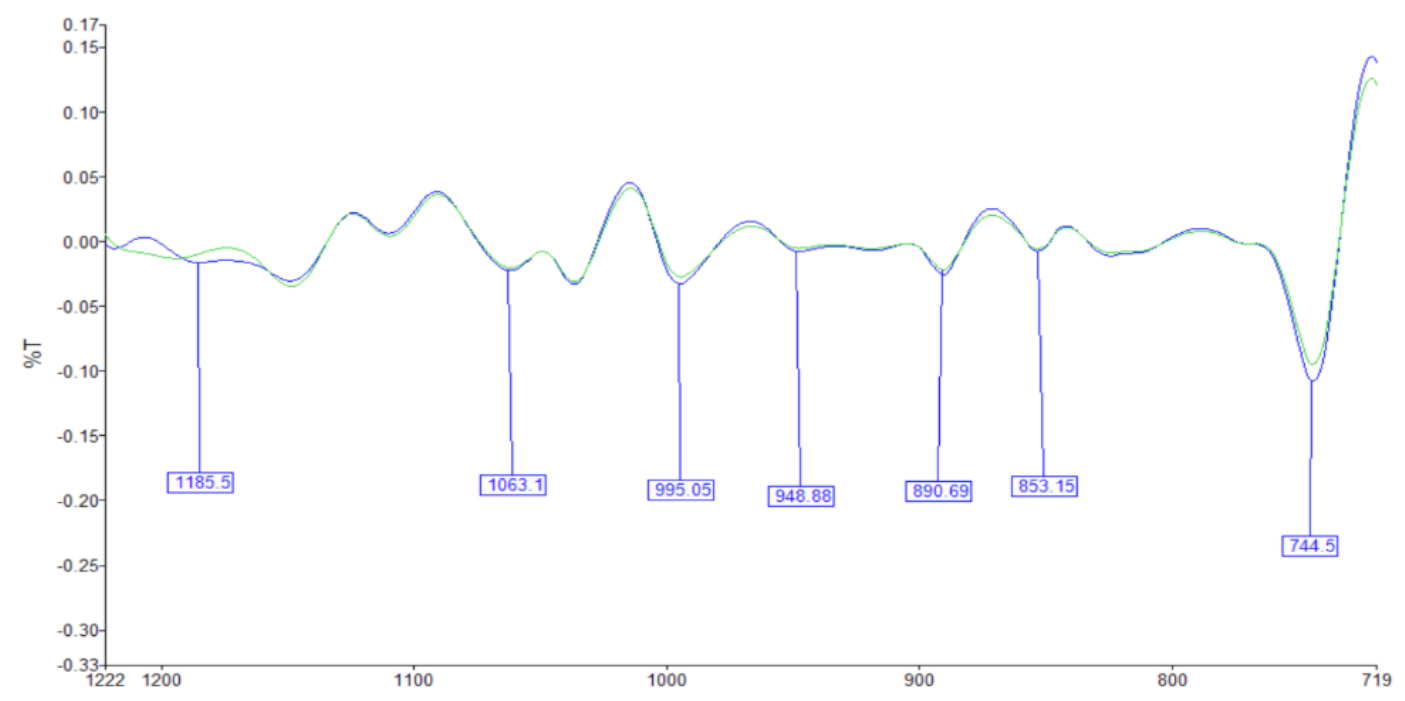

Figure 5. FTIR Spectra of SHF finished polyester fabric in regions Si-O-Si and C-F peaks

Water repellency spray test was performed on the SHF finish treated samples using AATCC TM22 (ISO 4920) that yielded a rating of 90 according to the AATC rating chart. It was observed that the treated sample exhibited a slight random sticking of water on the upper surface, which was removed by shaking the fabric sample, and there was no water mark at all on the lower side of the
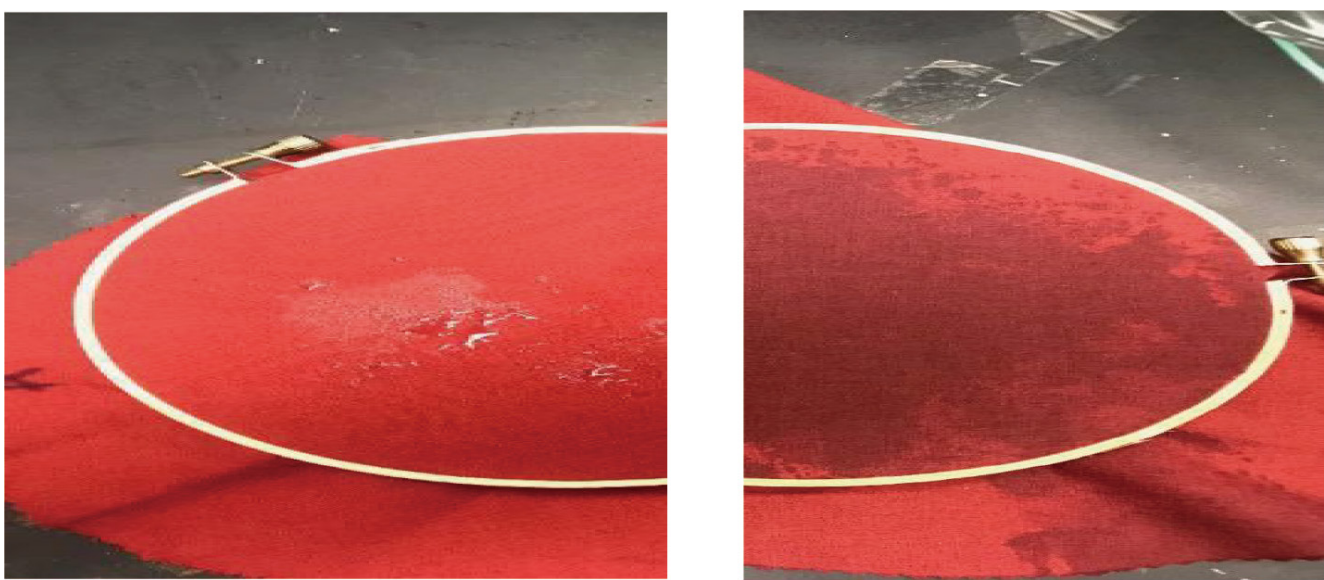

Figure 6. FS-treated sample and original untreated sample after AATCC TM22 water spray test

A stain repellency test was conducted by using the AATCC 130 method, and the rating was given according to the Stain Release Replica grade (AATCC 130). The original untreated and FS-treated samples were stained with mayonnaise, jam, and hot sauce. The samples were left for 30 minutes after application on the surface, after which the applied staining agents were wiped out by a dry tissue paper. As seen in Figure 7, there was no stain or sample but original untreated sample was completely wet on both upper and lower surface after the water spray, obtaining the lowest rating of 0 . Usually a rating of 80 rating is considered in the industry as a requirement for a superhydrophobic fabric. A test sample is illustrated in figure 6. any mark on the SHF-treated superhydrophobic specimen, however a clear stain was noticed on the original untreated sample. According to the AATCC 130 stain release replica grading chart, the SHF-treated sample received a grade of 5 , which indicates there was no stain on it. Whereas the untreated fabric received a 0 rating, because there was no change in the stain created by the staining agents. 


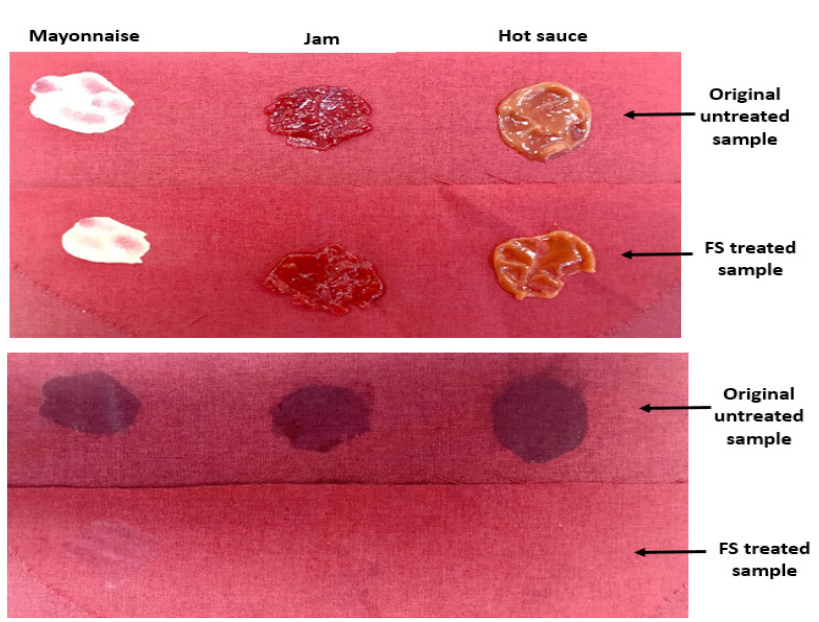

Figure 7B. original untreated and SHF-treated samples after wiping out with a tissue

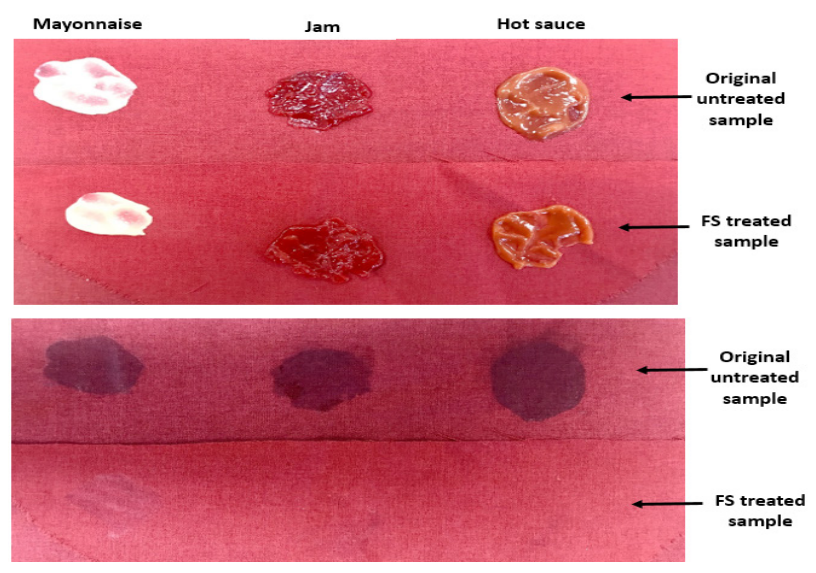

Figure 7A. The original untreated and SHF-treated samples after the applied staining agents

The SHF finished fabric was tested against coffee heated to the temperature at which it is generally served at coffee shops $\left(60^{\circ} \mathrm{C}\right)$, Coke at $10^{\circ} \mathrm{C}$, which is the normal temperature inside refrigerators in stores, and red wine at room temperature $\left(25^{\circ} \mathrm{C}\right)$. After these liquids were poured on to the fabric, they were observed to form spherical droplets as shown in Figure 8. After 10 minutes, when the fabric was tilted, and the liquid drops were observed to roll out without wetting or staining the fabric. When the droplets were standing on the fabric, the contact angle (CA) values were measured to be above $140^{\circ}$, which is in the range of superhydrophobic character as given in Figure 8. Furthermore, vegetable cooking oil drops were placed on the fabric and was watched for one hour. The fabric did not absorb the oil at all, and after 1 hour, the oil drops were easily removed by wiping with dry tissue paper, without any staining or absorption of the oil in the fabric.

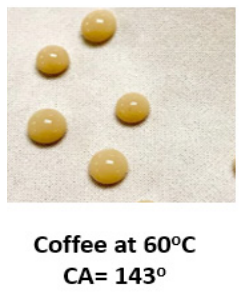

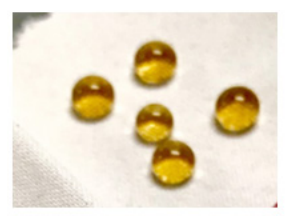

Coke at $10^{\circ} \mathrm{C}$ $\mathrm{CA}=161^{\circ}$

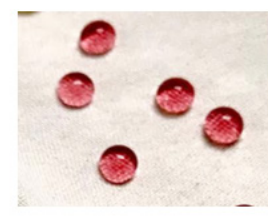

Red Wine at $25^{\circ} \mathrm{C}$ $\mathrm{CA}=1 \mathbf{1 4}^{\circ}$
Figure 8. illustrating liquid drops on the SHF finish treated polyester fabric surface

\section{Conclusions}

This study developed a Super-hydrophobic PET polyester fabric using a specific SHF finish. The treated fabric did not show any changes in the surface appearance and in tactile property. Experimental data showed that superhydrophobic property of the fabric was maintained even after undergoing 50,000 abrasion cycles. Different kinds of liquids including tea, coffee and wine, formed spherical droplets on the fabric surface and rolled off the fabric surface without wetting or adhering to the fabric. The treated fabric had a water contact angle $>=170^{\circ}$, even after 50,000 abrasion cycles, implying the finish was durable. Water Repellency Spray test provided 90 (AATCC Rating) which is the commonly accepted value by industry for a super-hydrophobic fabric. No residue and stain were observed on the wiped fabric during the stainrepellency test against mayonnaise, jam and hot sauce. FTIR data identified the presence of SHF finish on both the treated fabric before and after abrasion. It is concluded from the experimental data that the SHF finish used on the polyester fabric provided a durable super hydrophobic characteristic. A unique feature of this finish and the method for the super-hydrophobic fabric is its suitability for the commercial process and reasonable cost. Superhydrophobic finished fabrics have several applications including apparel, automotive interior and hood for the convertible automobiles, army clothing, medical textiles, military clothing, furniture, outdoor furniture and other.

\section{References}

[1] Zhao H., Ras H.A. Definitions for Hydrophilicity, Hydrophobicity, and Superhydrophobicity: Getting the Basics Right. The Journal of Physical Chemistry Letters, 2014: 686-688.

[2] Barthlott W., Neinhuis C. Purity of the sacred lotus, or escape from contamination in biological surfaces. Planta, 1997: 1-8.

[3] Kawai A., Nagata H. Wetting Behavior of Liquid on Geometrical Rough formed by Photolithography, 
Journal of Applied Physics, 1994.

[4] Zhang X., Zhang J., Ren Z., Li X., Zhang X., Difu Z., Wang T., Tian T., Yang B. Modulating two-dimensional non-close-packed colloidal crystal arrays by deformable soft lithography. Langmuir, 2009.

[5] Chen J. K, Qui J. Q., Fan S. K., Kuo S. W., Ko F. H., Chih- Wei C., Chang F. C. Using Colloid Lithography to fabricate silicon nanopillar arrays on silicon substrates. Journal of Colloid and Interface Science, 2012.

[6] Polizos G., Tuncer E., Qiu X., Aytug T., Michelle Kidder K., Messman J. M, Sauers I. From Hydrophobic to Superhydrophobic and Super hydrophilic Siloxanes by Thermal Treatment. Langmuir, 2011.

[7] Bao X.M., Cui J.F., Sun H.X., Liang W.D. Zhu Z.Q., An J., Yang B.P., La P.Q., Li A. Facile preparation of super-hydrophobic surfaces based on metal oxide nanoparticles. Applied Surface Science, 2014: 473480.

[8] Sahoo B.N., Kandasubramanian B. Recent progress in fabrication and characterization of hierarchical biomimetic super-hydrophobic, Royal Society of Chemistry Advances, 2014.

[9] Hoofnagle's H.F.,Wu D.,With G.D., Ming W. Biomimetic Superhydrophobic and Highly Oleophobic Cotton Textiles, Langmuir, 2007.

[10] Ivanova N.A., Lavretsky A.K., Ivanova N.A., Zaretskaya A.K. Imparting Waterproof Properties to Cotton
Surface. Protection of Metals and Physical Chemistry of Surfaces, 2011: 369-371.

[11] Xu C.H., Jia S.T., Zhang J., Tian L.Q. Super-hydrophobic surfaces on cotton textiles by complex coating of silica nanoparticles and hydrophobization, Thin Solid Films, 2009.

[12] Das I., De G. Zirconium based super-hydrophobic coatings on cotton fabrics exhibiting excellent durability for versatile use, Scientific Reports, 2015: 1-11.

[13] Meng S., Ye Y., Mansouri J.,Chen V. Crystallization behavior of salts during membrane distillation with hydrophobic and super-hydrophobic capillary membranes. Journal of Membrane Science, 2015: 165176.

[14] Bendak, S. M., \& Marsafi, E. Effects of chemical modifications on polyester fibers. Journal of Islamic Academy of Sciences, 1991, 4(4): 275-284.

[15] Río, O. I., Kwok, D.Y., Wu, J. R., Alvarez, J. M., Neumann, A.W. Contact angle measurements by axisymmetric drop shape analysis and an automated polynomial fit program. Colloids and Surfaces A: Physicochemical and Engineering Aspects, 1998, 143(2-3): 197-210.

[16] Brassard, J. D, Sarkar D. K., Perron, J. Fluorine based superhydrophobic coatings. Applied Sciences, 2012, 2(2): 453-464. 